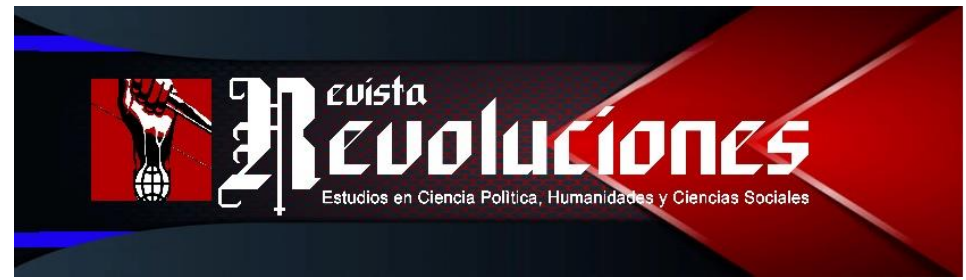

ARTÍCULO ORIGINAL

\title{
PROPUESTA METODOLÓGICA: INTEGRACIÓN DE LAS TECNOLOGÍAS DE LA INFORMACIÓN Y LA COMUNICACIÓN AL PROCESO DE EDUCOMUNICACIÓN
}

\author{
Methodological proposal: integration of information and \\ communication technologies to the educommunication process
}

\section{Fredy Eduardo León}

Martínez ${ }^{1}$

UNIVERSIDAD CATÓLICA DE CUENCA. ECUADOR

https://orcid.org/oooo-ooo1-

6811-1135
Fernando Mauricio

León Martínez

UNIVERSIDAD CATÓLICA DE CUENCA. ECUADOR

https://orcid.org/o0oo-00023969-2986
Victor Hugo Guillermo

Ríos

UNIVERSIDAD CATÓLICA DE CUENCA. ECUADOR

https://orcid.org/oooo-0003$3637-4453$

DOI: https://doi.org/10.35622/j.rr.2021.04.004

Recibido: 03-VII-2020 / Aceptado: 13-VII-2021 / Actualizado: 06-VIII-2021

\section{Resumen}

El objetivo fue diagnosticar el equipamiento y el uso de las TIC's en el proceso de enseñanza y aprendizaje en los docentes y estudiantes de la Carrera de Ciencias de la Información y Comunicación Social de la Universidad Católica de Cuenta (Ecuador). El enfoque de la investigación empleado fue de corte mixto (cualitativo-cuantitativo), de tipo descriptivo y diseño transversal. La población estuvo conformada por 10 docentes (entre 40 a 53 años) y 103 estudiantes del primero al décimo ciclo (entre 17 a 36 años). Los instrumentos para la obtención de datos fue el cuestionario de tipo cerrado y establecido en escala de Likert y como complemento fue la guía de observación, ambos estructurados en tres componentes: 1) Estructura y uso de la tecnología. 2) Percepciones sobre el uso de las herramientas tecnológicas y 3) Equipamiento tecnológico, su obtención de información se generó en base a un rastreo y análisis bibliográfico en buscadores académicos, revistas, congresos y seminarios; así como también en bases de datos científicas, que permitió proporcionar un análisis en los elementos indispensables para el estudio de la investigación, donde se determina que los estudiantes tienen en un mayor uso de las tecnologías de la comunicación en relación al de sus docentes, puesto que los estudiantes al estar en un sistema 'millennials' tienen un mejor conocimiento y destrezas, ya que en su generación de los ochenta estos cuentan con un acto grado de conocimiento tecnológico valores sociales y éticos.

${ }^{1}$ Correspondencia: fleduardomartinez@gmail.com

ISSN: 2710-0499 ISSN-L: 2710-0480

Esta obra está bajo una licencia internacional Creative Commons Atribución 4.0.

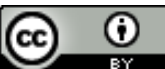


Palabras Clave: Alternativas metodológicas, comunicación, educomunicación, tecnología.

\section{Abstract}

The purpose was to diagnose the equipment and the use of ICTs in the teaching and learning process in teachers and students of the Information Sciences and Social Communication Career of the Catholic University of Cuenta (Ecuador). The research approach used was qualitative-quantitative, descriptive and cross-sectional. The sample population consisted of 10 teachers (between 40 and 53 years old) and 103 students from the first to the tenth cycle (between 17 and 36 years old). The instruments for obtaining data was the closed questionnaire established on the Likert scale and as a complement it was the observation guide, both structured in three components: 1) Structure and use of technology. 2) Perceptions on the use of technological tools and 3) Technological equipment, their obtaining of information was generated based on a bibliographic tracking and analysis in academic search engines, magazines, conferences and seminars; as well as in scientific databases, which allowed to provide an analysis of the essential elements for the study of research, where it is determined that students have a greater use of communication technologies in relation to that of their teachers, since that students, being in a millennial system, have better knowledge and skills, since in their generation of the eighties they have an act of technological knowledge, social and ethical values.

Keyword: Methodological alternatives, communication, educommunication, technology.

\section{INTRODUCCIÓN}

La educación no puede existir sin comunicación, la modificación colectiva en la educación no se impartiría si no existe el diálogo. La comunicación es la formación de un sujeto crítico que interactúa con los procesos comunicacionales permitiendo codificar e interactuar con los avances tecnológicos, incluyendo los medios masivos como parte activa del proceso (Huergo, 2017; Pérez, 1974). Toda transformación humana y social es consecuencia de la dialogicidad (Ramón, 2019).

Por eso creemos que la comunicación horizontal o participativa es el único proceso capaz de realizar al hombre y de llevarlo a la creación y al compromiso. Para permitir esa situación dinámica, de evolución constante, es indispensable la comunicación dialógica que origina el pensar crítico (Pérez, 1974).

En la era de la comunicación y la información el aprendizaje será la base para el desarrollo, el crecimiento y el progreso de la sociedad y ofrece soluciones a problemas de distinta naturaleza a través de la creación, acceso, manejo e intercambio de contenido electrónico. Por lo tanto, el sistema educativo debe favorecer la formación continua de los docentes para desarrollo de sus competencias lingüísticas en cada uno de los niveles educativos, a fin de responder a las necesidades, los intereses y los retos de la población estudiantil (Ricardo Barreto \& Iriarte Díazgranados, 2017). 
Los estudios muestran que la desmotivación y falta de interés del estudiante es un problema que se ve reflejado en los jóvenes al recibir las clases por sus docentes, se encuentran sobrecargados con información y no utilizan adecuadamente la tecnología.

En consideración de acuerdo al estudio analizado sobre el proceso continuo y de transformación lleva a un diagnóstico preliminar para la investigación cuyo objetivo fue elaborar una estrategia metodológica que permita formar un sujeto crítico que interactúe con los procesos comunicacionales codificando e interactuando con los avances tecnológicos, incluyendo los medios masivos como parte activa del proceso pedagógico por esta razón la comunicación y cognición son conceptos que no pueden separarse, de allí el término educomunicar. Kaplún (2002) afirma: "cuando el sujeto educando logra expresar una idea de modo que otros puedan comprenderla es cuando él mismo la aprende y la comprende verdaderamente" (p. 230). Esta realidad no es ajena de acuerdo al análisis de la investigación realizada, por cuanto al fortalecer a sus docentes y estudiantes mediante la integración de las Tics implica la integración de dos disciplinas: la educación y la comunicación siendo que estas deben ir combinadas con la práctica a través de los medios de comunicación existentes, por lo tanto, la educomunicación se constituye en una participación en el proceso comunicativo que son esenciales y se centran en el espacio escolar, espacio de la clase, como se maneja la comunicación entre docentes y estudiantes, cual es el contenido de los mensajes, los canales por los que fluye y asociado a ese espacio se puede mencionar el uso de la tecnología y la comunicación dando paso a la práctica basadas en el dialogo y sus aptitudes y sus concepciones pedagógicas (Ezequiel et al., 2019).

Por lo expuesto, en esta investigación se planteó como objetivo encontrar un proceso de avances educativos, tecnológicos, informativos y comunicativos que incidan y regulen los tradicionales y modernos modos de transferir saberes por parte del docente al estudiante, de una manera sustancial en cuanto a su estructura, forma de planificar y desarrollar clases, gestión y administración académica, como también la investigación y difusión del conocimiento.

\section{MÉTODO Y MATERIALES}

La investigación desarrollada en el contexto integración de las TIC al proceso educomunicativo, es de corte cuali-cuantitativo. Se basa más en una lógica y proceso inductivo que va de lo particular a lo general.

La estrategia que se propuso en el trabajo de investigación está conformada por las etapas de: diagnóstico, planificación, ejecución, instrumentación y evaluación. En cada una de las etapas a desarrollarse se anotan acciones con objetivos, responsables, participantes, formas de evaluación y plazos para la realización de las mismas. Se usó como técnica la encuesta y observación mediante la percepción directa de los elementos del objeto estudiado. 
Esto significa que, el punto de partida se sitúa el campo de comportamientos manifiestos: el alumno responde a las estimulaciones de la comunicación y del medio a través de una sucesión de comportamientos verbales y no verbales que es la base de la observación directa del educador (Ketele, 1984).

Las técnicas a realizadas fueron:

- Encuesta a los docentes sobre el conocimiento de las TICs y el uso de las mismas en el aula de clase.

- Encuesta a estudiantes de la Unidad Académica de Ciencias Sociales, Periodismo, Información y Derecho de la Universidad Católica de Cuenca determinando el nivel de conocimientos que tiene el estudiante sobre las TIC, con el que poseen los profesores de la Institución.

- Encuesta sobre la inserción de algunas de las tecnologías de la Información y la Comunicación.

- Encuesta dirigida a los estudiantes sobre lo que ellos consideran motivante.

Para ello se estudió lo que la gente, piensa, dice y hace con respecto a determinada situación, en nuestro caso el uso de las TICs en los procesos educativos; desde este enfoque la participación activa de los actores involucrados es clave en el proceso de cambio de mentalidad y acción de estas tecnologías. Mediante este tipo de investigación se logró conocer las relaciones sociales entre las personas y a partir de ello se analiza la situación que se presenta desde el proceso de investigación.

El total de docentes encuestados fueron 10, género masculino, con un promedio de edad de 50,3 años. El total de estudiantes encuestados fueron 93, entre 17 y 49 años de edad con un promedio de 22,53 años, divididos en 56 del género masculino con un rango de edad entre 17 y 49 años, con un promedio 23,39 años, y 37 del género femenino, con edades entre 18 y 32 años con un promedio de 21,24 años.

\section{RESULTADOS}

¿Qué elementos podrían integrar una propuesta metodológica que fortalezca la integración de las Tics en los procesos educomunicativos en la enseñanza aprendizaje? Participación del Docente: A través del cuestionario realizado a los docentes sobre aspectos que motiven a desarrollar su labor en el aula de clases estos fueron los resultados.

Tabla 1. Respuestas de los Docentes

\begin{tabular}{|c|c|c|c|c|c|}
\hline $\begin{array}{c}\text { Herramientas } \\
\text { TIC }\end{array}$ & $\begin{array}{c}\text { Muy } \\
\text { Importante }\end{array}$ & Importante & $\begin{array}{c}\text { Medio } \\
\text { Importante }\end{array}$ & $\begin{array}{c}\text { Poco } \\
\text { Importante }\end{array}$ & $\begin{array}{c}\text { Nada } \\
\text { importante }\end{array}$ \\
\hline Computadora & $70 \%$ & $20 \%$ & & & $10 \%$ \\
\hline
\end{tabular}




\begin{tabular}{|c|c|c|c|c|c|}
\hline $\begin{array}{l}\text { Proyectores } \\
\text { Multimedia }\end{array}$ & $80 \%$ & & $10 \%$ & $10 \%$ & \\
\hline Pizarras Digitales & $40 \%$ & $10 \%$ & $10 \%$ & $10 \%$ & $30 \%$ \\
\hline $\begin{array}{c}\text { Archivos digitales } \\
\text { portátiles }\end{array}$ & $40 \%$ & $20 \%$ & $10 \%$ & $20 \%$ & $10 \%$ \\
\hline $\begin{array}{l}\text { Bibliotecas } \\
\text { virtuales }\end{array}$ & $60 \%$ & $20 \%$ & $10 \%$ & & $10 \%$ \\
\hline
\end{tabular}

Fuente: Docentes Universidad Católica de Cuenca

En la tabla 1, las respuestas de los docentes en un promedio del $70 \%$ se dirigen hacia la computadora, proyectores multimedia y bibliotecas virtuales, como muy importantes herramientas TIC, que favorecen al desarrollo de las labores dentro del aula de clase; al ser una tecnología que se encuentra al alcance de las universidades, docentes y estudiantes. Mientras que un $50 \%$ divide la categorización entre muy importante y poco importante y nada importante el uso de pizarras digitales o archivos digitales portátiles al momento de impartir sus materias, a pesar de los resultados no podemos desechar estas herramientas tecnológicas que en su momento y al ser un recurso disponible, ayudaran al proceso educomunicativo.

De los resultados obtenidos y apegado a la hipótesis planteada, si mejora la metodología que permite la integración de las TICs se fortalece el proceso educomunicativo en la Carrera de Ciencias de la Información y Comunicación Social de la Universidad Católica de Cuenca, el proceso educomunicativo se usa apropiada y organizadamente, motivando mediante capacitaciones dirigidas un correcto uso de las herramientas TICs disponibles, en el proceso de enseñanza-aprendizaje.

Participación del Estudiante: Se realizó una encuesta a los estudiantes obteniendo los siguientes resultados con respecto a la motivación del desarrollo en su proceso académico utilizando las TIC.

Tabla 2. Respuesta de los Estudiantes

\begin{tabular}{lccccc}
\hline \multicolumn{1}{c}{$\begin{array}{c}\text { Herramientas } \\
\text { TIC }\end{array}$} & $\begin{array}{c}\text { Muy } \\
\text { Importante }\end{array}$ & Importante & $\begin{array}{c}\text { Medio } \\
\text { Importante }\end{array}$ & $\begin{array}{c}\text { Poco } \\
\text { Importante }\end{array}$ & $\begin{array}{c}\text { Nada } \\
\text { importante }\end{array}$ \\
\hline $\begin{array}{l}\text { Computadora } \\
\text { Plataformas }\end{array}$ & $76,34 \%$ & $12,90 \%$ & $3,23 \%$ & $1,08 \%$ & $6,45 \%$ \\
$\begin{array}{l}\text { Educativas } \\
\text { Virtuales }\end{array}$ & $56,99 \%$ & $32,25 \%$ & $6,45 \%$ & $3,23 \%$ & $1,08 \%$ \\
$\begin{array}{l}\text { Redes Sociales } \\
\text { Proyectores }\end{array}$ & $36,57 \%$ & $32,25 \%$ & $19,35 \%$ & $5,38 \%$ & $6,45 \%$ \\
$\begin{array}{l}\text { Multimedia } \\
\text { Pizarras Digitales }\end{array}$ & $49,45 \%$ & $30,11 \%$ & $8,60 \%$ & $2,15 \%$ & $9,68 \%$ \\
$\begin{array}{l}\text { Archivos Digitales } \\
\text { Portátiles }\end{array}$ & $62,36 \%$ & $12,90 \%$ & $9,68 \%$ & $5,38 \%$ & $9,68 \%$ \\
$\begin{array}{l}\text { Bibliotecas } \\
\text { Virtuales }\end{array}$ & $54,84 \%$ & $26,88 \%$ & $7,53 \%$ & $2,15 \%$ & $8,60 \%$ \\
\hline
\end{tabular}

Fuente: Estudiantes Universidad Católica de Cuenca 
En la tabla 2 se presenta los aspectos que le motivan al estudiante a desarrollar mejor su proceso académico utilizando las tecnologías de la información y comunicación, las respuestas de los estudiantes en un promedio del 80 \% se dirigen hacia la herramienta TICs como muy importantes e importantes, que favorecen al desarrollo de las actividades académicas dentro del aula de clase; al ser una tecnología que se encuentra al alcance de las universidades, docentes y estudiantes. Mientras que un 20 \% divide la categorización entre medio importante y nada importante, a pesar de los resultados no podemos desechar estas herramientas tecnológicas que en su momento y al ser un recurso disponible, aportaran al proceso educomunicativo.

Se realizó una entrevista a los docentes de la carrera obteniendo los siguientes comentarios con respecto a la siguiente pregunta ¿Qué recursos tecnológicos recomendaría usted para mejorar el proceso de enseñanza-aprendizaje?

El docente contesta lo siguiente: Se destaca la transmedialidad que existe al momento del proceso educomunicativo con las TICs, con una adecuada capacitación y conciencia por parte de docentes y estudiantes. Es importante la convergencia de lo digital y lo convencional, la tecnología y el contacto interpersonal, docente-estudiante. Apoyarse adecuadamente en la plataforma virtual, para foros, debates, etc. Sugerir un mayor ancho de banda inalámbrico para aprovechar las ventajas del internet, en las bibliotecas virtuales.

Tabla 3. Equipamiento Tecnológico

\begin{tabular}{|c|c|c|c|c|c|}
\hline Pregunta & $\begin{array}{l}\text { Muy de } \\
\text { acuerdo }\end{array}$ & $\begin{array}{c}\text { De } \\
\text { acuerdo }\end{array}$ & $\begin{array}{c}\text { Ni de } \\
\text { acuerdo, ni } \\
\text { en } \\
\text { desacuerdo }\end{array}$ & $\begin{array}{c}\text { En } \\
\text { desacuerdo }\end{array}$ & $\begin{array}{c}\text { Muy en } \\
\text { desacuerdo }\end{array}$ \\
\hline $\begin{array}{l}\text { 1.- Considera que la } \\
\text { institución educativa } \\
\text { donde estudia debe } \\
\text { proporcionar una } \\
\text { computadora para sus } \\
\text { actividades académicas. }\end{array}$ & $78,49 \%$ & $18,28 \%$ & $3,23 \%$ & & \\
\hline $\begin{array}{l}\text { 2.- Considera que la } \\
\text { institución educativa } \\
\text { cuenta con equipos y } \\
\text { medios tecnologicos } \\
\text { necesarios que fortalecen } \\
\text { el proceso } \\
\text { educomunicativo. }\end{array}$ & $10,75 \%$ & $41,94 \%$ & $31,18 \%$ & $9,68 \%$ & $6,45 \%$ \\
\hline $\begin{array}{l}\text { 3.- Considera que la } \\
\text { institución educativa } \\
\text { cuenta con adecuados } \\
\text { medios pedagógicos que } \\
\text { garanticen los procesos } \\
\text { educomunicativos de } \\
\text { enseñanza-aprendizaje. }\end{array}$ & $17,20 \%$ & $51,61 \%$ & $20,43 \%$ & $7,53 \%$ & $3,23 \%$ \\
\hline $\begin{array}{l}\text { 4.- La educomunicación } \\
\text { que le brinda la institución } \\
\text { educativa, en lo académico } \\
\text { e intelectual, cumple con } \\
\text { sus expectativas. }\end{array}$ & $21,51 \%$ & $41,94 \%$ & $21,51 \%$ & $10,74 \%$ & $4,30 \%$ \\
\hline
\end{tabular}




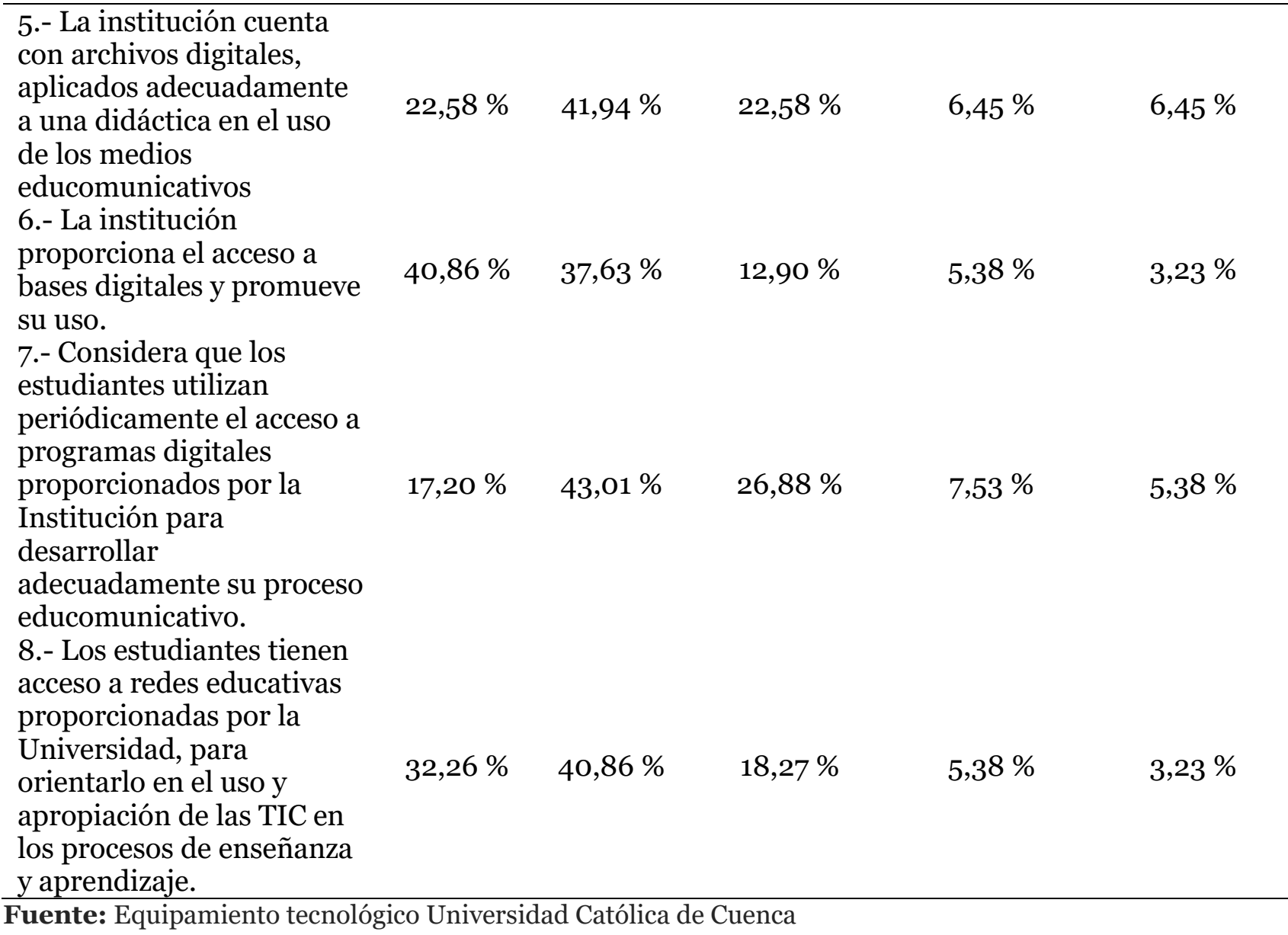

De las respuestas, se puede dar una lectura general de que aproximadamente el $70 \%$ de los encuestados, esta entre el muy de acuerdo y de acuerdo en que la Universidad debe de proveer de los medios tecnológicos, didácticos y pedagógicos necesarios para impartir su catedra, en relación directa con el proceso educomunicativo de enseñanza-aprendizaje, además se destaca el mejor el uso de la plataforma educativa, así como la motivación que se debe tener para orientar el uso educativo-investigativo de las diferentes redes educativas.

Sin embargo, es importante destacar que un $20 \%$ se ubica entre ni de acuerdo ni en desacuerdo y en desacuerdo, esto se nota claramente en las preguntas, que, a pesar de ser un porcentaje relativamente bajo, estas tienen relación directa con las capacitaciones que proporciona la universidad para mejorar el manejo y buen uso de las TICs al momento de desarrollar los procesos educomunicativos, especialmente en materia de investigación, apoyado en redes o plataformas científicas. El $10 \%$ restante se ubica en desacuerdo y muy en desacuerdo, ante las necesidades educomunicativas en uso de las TICs.

\section{DISCUSIÓN}

Integración curricular de TICs es el proceso de hacerlas enteramente parte del curriculum, como parte de un todo, permeándolas con los principios educativos y la didáctica que conforman el 
engranaje del aprender. Ello fundamentalmente implica un uso armónico y funcional para un propósito del aprender específico en un dominio o una disciplina curricular (Sánchez, 2003), de tal manera que: al incorporar las tecnologías de la información y comunicación (TIC) en el aula sea un proceso que no se limite el contar con las herramientas que conforman las tecnologías, sino que lo más importante es construir un uso educativo y didáctico de las mismas así consolida un aprendizaje significativo en base a la tecnología (Díaz-Barriga, 2013).

Por esta razón el uso de las tecnologías permite al docente como al estudiante desempeñar papeles enfocados más a la construcción y a la investigación sobre la información; todo ello sin olvidarnos de que la figura fundamental en el proceso de enseñanza aprendizaje es el docente y no las herramientas tecnológicas que se utilicen (Díaz, 2019).

Por otra parte, el integrar las TIC al proceso educomunicativo para volverla parte vital de los procesos de gestión educativa, deben controlarse su uso de alguna manera, por cuanto los estudiantes pueden volverse dependientes totalmente de la tecnología sin prestar atención a otros elementos tan importantes como son las explicaciones del docente en ciertos puntos de una asignatura que así lo requieren; en algunos casos, los aprendices, desafortunadamente dedican casi siempre su tiempo a la búsqueda de la información en la web, información que en un alto porcentaje no es cierta o distorsionada y no consultan libros serios, de autores reconocidos o en su defecto, acudir a las tutorías que los centros educativos prestan para aquellos que no asimilen los temas de forma clara dentro del aula de clases (Velandia, Gómez \& Bello, 2018).

Permitiendo que el docente sea un formulador de la enseñanza que pueda elegir o diseñar correctamente las estrategias de acuerdo al contexto del alumno para que el estudiante logre alcanzar un aprendizaje significativo, debemos mencionar que el proceso educacional deberá manejarse en el contexto comunicacional como formas de entrega de información con el compromiso de generar conceptos de autoformación colectiva sustentada en realidades discernidas a partir de significaciones recibidas y las múltiples interacciones derivadas de estas. En estos momentos en los que se habla de calidad si queremos ofrecer una enseñanza universitaria basada en estrategias que propulse el consumo de TICs, esta deben ser apoyada en el 'learning by doing' alejada de la concepción actual de 'teaching by telling' (Marín et al., 2009) El alumno ha de poder disfrutar de la experiencia de aprender teniendo un papel protagonista, sintiéndose con la responsabilidad de su propio aprendizaje y poniendo en práctica sobre la misma realidad todo aquello sobre lo que genera conocimiento. Es decir, la metodología que escojamos debe facilitar el apodado learning by doing, o aprender haciendo (López-García, 2016).

Es importante mencionar que el aprendizaje basado en esta metodología activa que se incluye dentro del enfoque del constructivismo del aprendizaje, en la que cada alumno construye su propio conocimiento y elabora sus contenidos desde la interacción que se produce en el aula (Iborra Cuéllar \& Izquierdo Alonso, 2010, p. 3). 
Permite que, se potencie un espíritu emprendedor y una autonomía, tomando decisiones y valorando los distintos puntos de vista y perspectivas de otros compañeros. El aprendizaje colaborativo posibilita interacciones entre individuos, en las que los alumnos se comunican, expresan y desarrollan un pensamiento crítico (Sáez Lopez \& Ruiz Ruiz, 2012), por ello, el pensamiento crítico es ese modo de pensar - sobre cualquier tema, contenido o problema - en el cual el pensante mejora la calidad de su pensamiento al apoderarse de las estructuras inherentes del acto de pensar y al someterlas a estándares intelectuales (Paul \& Elder, 2003). Por lo tanto, uno de los puntos importantes para la educomunicación, es generar en las personas un pensamiento crítico y autónomo, empleando para ello todos los medios de comunicación disponibles que desarrollen la creatividad crítica en el proceso de enseñanza-aprendizaje.

Por otro lado, el profesor universitario no sólo debe estar al día de los descubrimientos en su campo de estudio. Al mismo tiempo, debe también atender a las posibilidades de las TIC y a las eventuales innovaciones en los procesos de enseñanza-aprendizaje (Salinas, 2004), el reto, desde el punto de vista educativo, no pasa por el «deslumbramiento tecnológico», sino por saber aprovechar y adaptar la tecnología existente para llevar los contenidos de calidad a un estadio superior, bien por su capacidad motivadora, su fomento del aprendizaje colaborativo o su apuesta por el cambio social, entre otras posibilidades (Tornero \& Tejedor, 2016).

De tal manera que, los docentes asuman que las clases de informática o el uso puntual del ordenador no es una práctica sustentadora de los importantes aprendizajes que sus alumnos tienen que llevar a cabo. Pero al oponer una enseñanza basada en medios tradicionales frente a una enseñanza basada en medios electrónicos, se apela de un modo implícito a una idealización de la enseñanza y del aprendizaje basado en modelos tradicionales, impidiéndose un análisis crítico sobre el alcance de la enseñanza basada en los medios de siempre (Sunkel, 2006).

La introducción de las TIC en el proceso de enseñanza-aprendizaje necesariamente provoca una transformación en rol del profesor con relación al papel que juegan estas en función de la docencia universitaria, sin lugar a dudas estamos en presencia de un cambio que promueve en los docentes el desarrollo de la innovación (Meneses \& Bucheli, 2019). De esta manera, un docente competente en el empleo de las TIC será aquel que sepa qué, cómo y cuándo enfrentar las situaciones que le plantea su labor educativa, siendo capaz de reaccionar ante la complejidad y la incertidumbre del acontecer cotidiano del aula (Velandia et al., 2018). De esta manera, si se quiere integrar las TIC al currículo escolar se debe basar en 5 componentes esenciales para su efectiva implementación. 


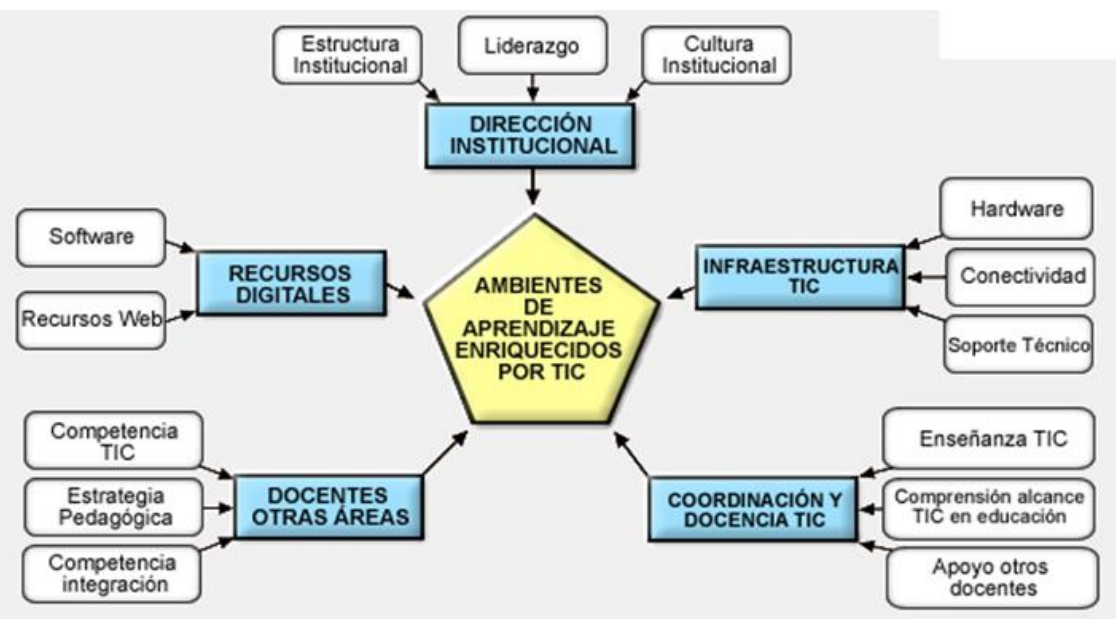

Figura 1: Componentes del modelo MITIC@

Fuente: EDUTEKA (2008). Modelo Mitic@: Modelo para Integrar las TIC al Currículo Escolar.

https://eduteka.icesi.edu.co/modulos/8/234/132/1

- Dirección Institucional: hace referencia al liderazgo administrativo, pedagógico y técnico requerido por parte de las directivas de la Institución Educativa y, a los cambios necesarios en su estructura y en su cultura organizacional;

- Infraestructura TIC: atiende los recursos tecnológicos propiamente dichos: hardware, software (sistema operativo y otras aplicaciones básicas), conectividad y soporte técnico;

- Coordinación y Docencia TIC: trata las funciones que deben desempeñar dentro de la Institución tanto el Coordinador Informático, como los docentes de esta asignatura;

- Docentes de otras Áreas: se refiere a las competencias que estos deben tener para poder integrar las TIC en la enseñanza de sus materias/asignaturas; y

- Recursos Digitales: atiende la disponibilidad y correcta utilización de software y recursos Web.

Siguiendo las investigaciones, puede decirse que, según la etapa de avance en la que se encuentran los proyectos de integración TIC en sus sistemas educativos, existen al menos tres grandes grupos de países (Zacarias, 2018):

- Países en Etapa de Integración: En éstos, las escuelas no solo cuentan con recursos tecnológicos, sino que se ha comenzado a capacitar a los docentes y se ha integrado el uso de las TIC en la currícula por ejemplo en América Latina, algunos de los países que se encuentran en esta etapa son: Chile, Uruguay, Argentina, México, Brasil, Costa Rica y Colombia

- Países en Etapa de Aplicación: En éstos, las autoridades de los sistemas educativos han comenzado a desarrollar experiencias piloto en ciertas escuelas elegidas, con resultados concretos. En América Latina, algunos de los países que se encuentran en esta etapa: El Salvador, Jamaica, Perú, República Dominicana y Trinidad y Tobago.

- Países en Etapa Emergente: En éstos, se ha tomado conciencia de los beneficios de incorporar las TIC en los sistemas educativos y presentan proyectos en fases iniciales, debido a diversas limitaciones. En América Latina, los siguientes países, entre otros, se encuentran en esta etapa: Guatemala y Paraguay. 
Por otra parte, es importante manifestar que la preparación de los docentes para la utilización de las TIC en el proceso de enseñanza aprendizaje. Según (Sunkel, 2006), los directivos exponen en la entrevista, que los docentes no poseen conocimientos suficientes para la aplicación de las TIC en el proceso de enseñanza y de aprendizaje, se limitan a aplicar algunos programas de informática, acceder a internet y buscar información, pero no están preparados para desarrollar recursos digitales y aprovechar las potencialidades de las TIC en el proceso de enseñanza aprendizaje de sus asignaturas a través de las Aulas Virtuales. Este es un elemento que se manifiesta en una gran cantidad de instituciones educativas del país y de América Latina.

\section{CONCLUSIONES}

La educomunicación integrada a las TICs es un campo de estudio y aplicación sumamente importante, especialmente por la relación directa de los medios de comunicación con nuestra profesión. Se destaca que los docentes no consideran significativo el uso de pizarras y archivos digitales portátiles al momento de impartir sus materias a pesar de que estas herramientas son indispensables para la transmisión de contenidos.

Si se mejora la metodología en la integración de las TIC estas favorecerán al proceso educomunicativo, para ello se debería motivar la capacitación en el uso correcto y adecuado de las tecnologías de información y comunicación.

Los factores transcendentales en la enseñanza aprendizaje es la convergencia entre lo analógico y lo digital, pues en este caso lo convencional y lo tecnológico mejora el contacto interpersonal docente-estudiante.

Es fundamental la transmedialidad que existe al momento del proceso educomunicativo con las TICs, con una adecuada capacitación y conciencia por parte de docentes y estudiantes y apoyarse adecuadamente en la plataforma virtual, para foros, debates, investigación e interacción con los medios, para esto es primordial considerar un mayor ancho de banda inalámbrica para aprovechar las ventajas del internet en las aulas y las bibliotecas virtuales y la predisposición de los docentes para su autoaprendizaje en los medios tecnológicos llegando así a impartir sus conocimientos y el buen uso de la educomunicacion a sus estudiantes.

\section{REFERENCIAS BIBLIOGRÁFICAS}

Díaz-Barriga, Á. (2013). TIC en el trabajo del aula. Impacto en la planeación didáctica. Revista Iberoamericana de Educación Superior. https://doi.org/10.22201/iisue.20072872e.2013.10.88

Díaz, R. P. (2019). Teaching digital competence in the teacher training institutes: Case of the Dominican Republic. Pixel-Bit, Revista de Medios y Educación, 55, 75-97. https://doi.org/10.12795/pixelbit.2019.i55.05

Ezequiel, M., Mendoza, B., Lagos, C. P., Jessenia, N., \& Morales, M. (2019). Ciudadanías digitales Perspectivas desde los medios, el Periodismo y la educomunicación editores. 
Huergo, J. A. (2017). Comunicación/Educación: Itinerarios transversales. In Comunicación Educación (pp. 3-25). https://doi.org/10.4000/books.sdh.183

Iborra Cuéllar, A., \& Izquierdo Alonso, M. (2010). ¿Cómo afrontar la evaluación del aprendizaje colaborativo? Una propuesta valorando el proceso, el contenido y el producto de la actividad grupal. Revista General de Información y Documentación, 20(1), 221-241.

Kaplún, M. (2002). Comunicador, Una pedagogía de la comunicación (el Popular).

Ketele, J. (1984). Observar para educar : observación y evaluación en la práctica educativas. 21.

López-García, C. (2016). Enseñar con TIC Nuevas y renovadas metodologías para la enseñanza Superior. Enseñar Con TIC Nuevas y Renovadas Metodologías Para La Enseñanza Superior, 9-16.

Marín, V., Asunción, M., \& López, R. (2009). No 35 Julio.

Meneses, K. L., \& Bucheli, M. G. V. (2019). El empleo de las TIC en la Educación Superior. Educando Para Educar, 20(37), 91-99.

Paul, R., \& Elder, D. L. (2003). La mini-guía para el Pensamiento crítico Conceptos y herramientas. Pensamiento, 26.

Pérez, F. G. (1974). Hacia una pedagogía basada en los medios de comunicación social: experiencias concretas en America Latina. CIEC.

Ramón, F. (2019). El aprendizaje dialógico: un paso más hacia la inclusión en el aula.

Ricardo, C., \& Iriarte, F. (2017). Las TIC en la Educación superior: experiencias de innovación. In Las Tic en educación superior.

Sáez Lopez, J. M., \& Ruiz Ruiz, J. M. (2012). Estrategias metodológicas, aprendizaje colaborativo y TIC: Un caso en la Escuela complutense latinoamericana. Revista Complutense de Educación, 23(1), 115-134. https://doi.org/10.5209/rev_RCED.2012.v23.n1.39105

Salinas, J. (2004). Teaching innovation and the use of ICT in university education. RUSC. Universities and Knowledge Society Journal, 1(1). https://doi.org/10.7238/rusc.vii1.228

Sanchez, J. (2003). Integración Curricular de TICs. Conceptos y modelos. Revista Enfoques Educacionales, 5(1), 51-65.

Sunkel, G. (2006). Las tecnologías de la información y la comunicación (TIC) en la educación en América Latina. Una exploración de indicadores.

Tornero, J. M. P., \& Tejedor, S. (2016). Ideas para aprender a aprender: manual de innovación educativa y tecnología. Editorial UOC.

Velandia, L. N. M., Gómez, L. A. P., Piragauta, J. D., Herrera, F. S., Aros, C. G., \& Bello, G. P. (2018). El papel de las tic en la transformación de la sociedad. In El papel de las tic en la transformación de la sociedad. https://doi.org/10.2307/j.ctv11wjdp

Velandia, L. N. M., Gómez, L. A. P., Piragauta, J. D., Herrera, F. S., Aros, C. G., Bello, G. P., \& Velandia, L. N. M. (2018). Las TIC en la educación. In El papel de las tic en la transformación de la sociedad (Vol. o, Issue o, pp. 11-32). https://doi.org/10.2307/j.ctv11wjdp.4

Zacarias, I. G. (2018). Las políticas de evaluación educativa en América Latina. Revista Fuentes, 20(2), 29-35. https://doi.org/10.12795/revistafuentes

Revista Revoluciones -67- Vol. 3, $\mathrm{N}^{\circ} 4$ (2021), pp. 56-67 